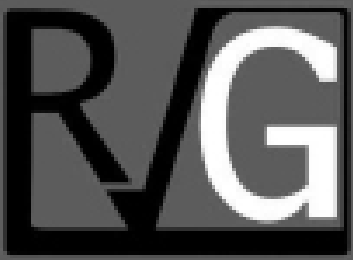

Año 24 No. 88

Octubre - Diciembre 2019

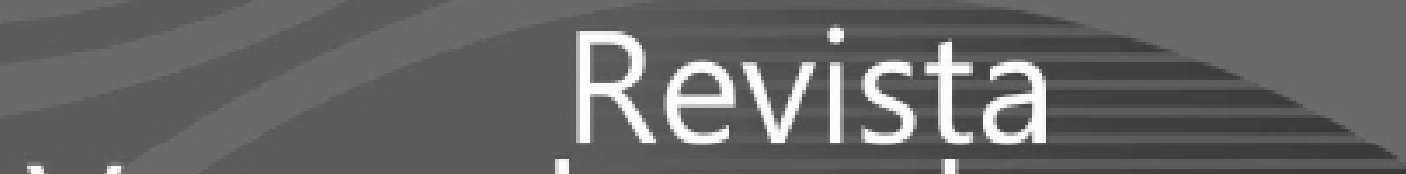

Venezolana de

verencla

UNIVERSIDAD DEL ZULIA (LUZ)

Facultad de Ciencias Económicas y Sociales

Centro de Estudios de la Empresa 


\title{
Producción de conocimiento teórico: Caso de Universidad Pedagógica Experimental Libertador (Venezuela)
}

\author{
Teresa de Jesús Molina Gutiérrez ${ }^{1}$ \\ Gisela Quintero² \\ Carlos Lizcano Chapeta ${ }^{3}$ \\ Lenin Horacio Burbano García ${ }^{4}$
}

\section{Resumen}

El objetivo del presente estudio fue caracterizar la producción de conocimiento teórico referencial de 10 tesis de posgrado presentadas en la Universidad Pedagógica Experimental Libertador (Venezuela). Se empleó la investigación cualitativa, complementada con el análisis del discurso (Van Dijk, 2005), el instrumento de recolección de datos fue una lista de cotejo, contentiva de 14 indicadores, estos se interpretaron mediante el análisis de contenido. Los resultados indican que, en general, hay dificultades para relacionar acertadamente los antecedentes con el problema, por lo que no se establece una perspectiva teórica de inicio. En cuanto a las bases teóricas predomina la transcripción de teorías sin la interpretación y comprensión necesarias, por lo que tampoco aparece la postura teórica que explicaría el objeto investigativo y serviría como apoyo para interpretar los hallazgos, en consecuencia, la respuesta a la problemática será insuficiente e inapropiada.

Palabras clave: investigación; trabajo de grado; marco teórico referencial; posgrado.

Recibido: 19-03-19 Aceptado: 19-06-19

1 PhD. Universidad Regional Autónoma de los Andes (UNIANDES) Ecuador:teresaj.molina@ gmail.com https://orcid.org/0000-0001-8341-0416

2 PhD. Universidad Nacional de Educación, Ecuador gishelinaq@hotmail.com https://orcid. org/0000-0002-3174-151X

3 Mg. Universidad Regional Autónoma de los Andes (UNIANDES) lizcha_4@hotmail.com https:// orcid.org/0000-0001-8349-6141

4 Mg Universidad Regional Autónoma de los Andes (UNIANDES), Ecuador: leninh_b@yahoo. com https://orcid.org/0000-0002-7115-4205 


\title{
Theoretical knowledge production: Case of the Libertador Experimental Pedagogical University (Venezuela)
}

\begin{abstract}
The objective of this study was to characterize the theoretical reference framework of 10 postgraduate theses presented at the Pedagogical Experimental University of Libertador (Venezuela). Qualitative research was used, complemented with Discourse Analysis (Van Dijk, 2005), the data collection instrument was a checklist, containing 14 indicators, these were interpreted through content analysis. The results indicate that, in general, there are difficulties in correctly relating the background to the problem, so that a theoretical starting point is not established. As for the theoretical basis, the transcription of theories predominates without the necessary interpretation and understanding, so the theoretical position that would explain the research object and serve as a support to interpret the findings does not appear, consequently, the answer to the problem will be insufficient and inappropriate.
\end{abstract}

Keywords: Research; undergraduate work; theoretical framework; postgraduate; knowledge; university.

\section{Introducción}

La necesidad de producir conocimientos científicos obliga a las universidades a optimizar sus esfuerzos al respecto, en este sentido la elaboración del Trabajo de Grado constituye la posibilidad de concretar esa exigencia investigativa, no obstante, el mismo es un género textual de gran complejidad metodológica y discursiva. Uno de sus componentes esenciales es el Marco Teórico Referencial, este involucra un proceso reflexivo que conduce a organizar los conocimientos vigentes sobre el interés investigativo y asumir una postura epistémica particular. Considerando esa relevancia

\section{La investigación científica} constituye la herramienta fundamental para la generación de conocimiento, es por ello que una de las responsabilidades de la educación superior es crear espacios adecuados para que los docentes y estudiantes se conviertan en los generadores de los saberes necesarios que transformen la realidad social.

Sin duda, no es una tarea sencilla, y la mayor evidencia de su dificultad está en la escasa productividad de muchas universidades latinoamericanas, razón por la cual se ha hecho una necesidad incorporar en las mallas curriculares la investigación como un eje transversal, así como convertirla en un criterio de evaluación institucional que se concreta en evidencias como proyectos, artículos, libros, participación en eventos de intercambio científico, entre otros.

Al respecto, la elaboración del trabajo de grado representa un requisito 
académico en la que el estudiante debe hacer converger los conocimientos teórico-prácticos adquiridos para resolver un problema propio de su área disciplinar. Dada la complejidad de su elaboración en la que intervienen tanto competencias textuales como competencias investigativas, resulta muy interesante analizar los procesos involucrados en su elaboración, en este caso, describir cómo se construye el marco teórico referencial, el cual representa el asidero teórico desde donde se explicarán los hallazgos encontrados una vez aplicados los métodos- del nivel empírico.

Se siguieron los procedimientos de la investigación cualitativa, complementada con el Análisis del Discurso (Van Dijk, 2005), para estudiar el discurso escrito como evento de comunicación social, cultural y cognitivo, en el que adquieren especial relevancia los aspectos textuales y contextuales de su producción.

El corpus lingüístico se integró por diez (10) tesis del área de Gerencia Educacional y Gestión Comunitaria, las cuales se registran con códigos para mantener la confidencialidad necesaria; se determinó como criterio de escogencia haberlas presentado en el período 2014-2017.

Como instrumento de recolección de los datos se empleó una lista de cotejo, contentiva de 14 indicadores, los mismos permitieron determinar las características de las bases teóricas en los trabajos de grado seleccionados.

Por otra parte, como técnica de interpretación de datos se usó el Análisis de Contenido; para ello se segmentó el corpus según criterios interpretativos y se escogieron categorías de análisis que permitieron describir, de modo exhaustivo, el texto correspondiente al marco teórico referencial

\section{Producción de conocimiento científico mediante la investigación}

La investigación constituye un proceso reflexivo, sistemático, controlado y crítico (Arias, 2012, Hernández, Fernández y Baptista, 2014) que valiéndose de la observación, experimentación y del conocimiento validado, persigue responder inquietudes para incrementar el conocimiento disciplinar. De modo que se convierte en el sustento de todas las actividades académicas en los distintos niveles educativos.

En la sociedad moderna la producción y sus correlatos, en este caso académicos, tiene una enorme importancia, reflejada en la exigencia a todas las universidades de cumplir con una de sus funciones básicas: la generación de conocimientos. Ello ha adquirido mayor vigencia en la actualidad pues se vive en la sociedad del conocimiento, en la que la universidad es por definición productora de principios, teorías, postulados, que deben enriquecer la episteme en la que se apoya la sociedad.

En opinión de Chacín, Cobos y Aray (2009), la investigación en el entorno universitario ha sido caracterizada como desarticulada, individualista, desvinculada, sin respuesta a las exigencias sociales, con poca preocupación por su producción y sin promoción para su aprovechamiento; desvirtuándose de esta manera, su concepción y una de sus funciones primordiales como es la participación activa en el proceso de desarrollo de las comunidades y del país. 
En lo que respecta al posgrado, es uno de los niveles formativos al que se le exige contribuir con la generación de conocimientos, sin embargo, los resultados obtenidos en muchos países latinoamericanos, no son del todo satisfactorios. Habría que agregar además que existen coincidencias (Erlich y Shiro, 2011, Bolívar y Beke, 2011), acerca de que el proceso investigativo es complejo y efectuarlo ofrece una serie de exigencias que ameritan una rigurosa preparación e interés.

Una de las actividades académicas, de obligatorio cumplimiento por parte de los estudiantes y que favorece la generación de nuevos saberes es la elaboración del Trabajo de Grado. Este es un género de escritura académica que exige una gran elaboración ya que la situación pragmática y discursiva en la que tiene lugar requiere de conocimientos disciplinares, lingüísticos y metodológicos muy específicos.

En este sentido, en este trabajo se asume que la investigación es clave para la producción de conocimiento teórico y es mediante la elaboración de trabajos de grado los medios primarios para generarlo, producirlo y generar procesos de transferencia, siendo este ultimo clave desarrollarlos desde las instituciones de educación superior.

\section{Experiencia de la Universidad Pedagógica Experimental Libertador (UPEL, Venezuela)}

En lo cuanto a la Universidad Pedagógica Experimental Libertador (UPEL, Venezuela), los trabajos de grado exigidos a los estudiantes en las especializaciones en Gerencia Educacional y Gestión Comunitaria, plantean como requerimiento elaborar una investigación aplicada que contenga un plan de intervención pedagógica para resolver problemas en contextos de aprendizaje, sin descuidar los requerimientos relativos al empleo adecuado de competencias investigativas y competencias lectoras y escritoras.

En general, todo el proceso investigativo reviste una gran importancia para el logro de las metas planteadas, pero el empleo del marco teórico referencial representa la herramienta que facilitará ubicar el problema en un contexto de relaciones con los conocimientos previos para favorecer su comprensión y explicación, lo cual tendrá una repercusión determinante en los hallazgos de nuevos saberes.

En este sentido, se requiere para la elaboración de las tesis de posgrado que el marco teórico referencial se estructure considerando los siguientes aspectos: estudios previos, bases teóricas y bases legales, asimismo, constituye el nicho teórico para la comprensión del interés investigativo. Teniendo en cuenta lo referido, el objetivo del presente estudio fue evaluar las características del marco teórico referencial en 10 trabajos correspondientes a las maestrías en Gerencia Educativa y Educación para la Gestión Comunitaria, de la UPEL, Núcleo Mérida, se reconoce la relevancia de las demás secciones de un trabajo de grado, sin embargo, al reconocer que la producción de conocimiento teórico surge en esta sección y crea condiciones de tipo científicas para abordar la realidad.

En cuanto a los referentes teóricos en los que se apoya esta investigación se debe precisar que el marco teórico referencial se define como un sistema coherente, armónico, lógico 
y coordinado de principios empíricos, históricos, metodológicos para el abordaje del problema, lógicamente interrelacionados. Asimismo, permite comprender la postura de distintos autores sobre el problema y la visión que asume el investigador para interpretar los resultados (Castañeda, 2014; Fidias, 2012; Reidil-Martínez, 2012; Sánchez, 2014).

Por su parte, Hernández, Fernández y Baptista (2014:60) lo llaman perspectiva teórica y la misma "proporciona una visión sobre dónde se sitúa el planteamiento propuesto dentro del campo de conocimiento en el cual nos "moveremos". Mientras que para Martínez (2009), en los estudios cualitativos, el compendio teórico, solo tiene como función servir de referencia para esclarecer el objeto de investigación.

Sin duda que la relevancia del marco teórico tiene que ver con: conocer los posibles errores en los que han incurrido otros estudios para evitar caer en los mismos, al establecer cómo se ha tratado previamente el problema investigativo (muestras, participantes, tipos de instrumentos, contextos, diseños) se obtiene una visión clara para determinar diferentes posibles perspectivas de abordaje, para precisar la delimitación del objeto de estudio y para escoger líneas de investigación novedosas.(Hernández, et. al, 2014). También se recopilan referencias con las cuales documentar el estudio, así como aporta contextos teóricos que facilitarán dar sentido a los datos empíricos. Definitivamente, luego de la detección de la literatura pertinente, de su análisis, interpretación, síntesis y comparación se debe estar en capacidad de adoptar una teoría mediante la cual se explicarán los hallazgos reportados.

En Cuadro 1, se resumen los resultados para cada categoría de análisis establecida.

\section{Cuadro 1}

\section{Categorías de análisis e interpretaciones}

\begin{tabular}{|c|c|}
\hline Categoría & Interpretación \\
\hline \multicolumn{2}{|l|}{ Antecedentes } \\
\hline $\begin{array}{l}\text { Se presentan reseñas de estudios relacionadas con } \\
\text { las variables de la investigación }\end{array}$ & Todos los trabajos responden a esta exigencia \\
\hline $\begin{array}{l}\text { Se establece clara vinculación y pertinencia entre los } \\
\text { antecedentes y el problema }\end{array}$ & $\begin{array}{l}\text { La mayoría de las tesis no establecen esta relación } \\
\text { con claridad }\end{array}$ \\
\hline $\begin{array}{l}\text { Los antecedentes son relevantes para el problema de } \\
\text { investigación }\end{array}$ & Todos los trabajos presentan antecedentes relevantes \\
\hline Las investigaciones presentadas son actualizadas & $\begin{array}{l}\text { La mayoría de las investigaciones responde a este } \\
\text { requerimiento }\end{array}$ \\
\hline \multicolumn{2}{|l|}{ Bases Teóricas } \\
\hline $\begin{array}{l}\text { El contenido de las teorías permite explicar el objeto } \\
\text { de estudio }\end{array}$ & $\begin{array}{l}\text { Seis trabajos no lo logran, los cuatro restantes si lo } \\
\text { hacen satisfactoriamente }\end{array}$ \\
\hline
\end{tabular}

Se evidencia análisis crítico de la información presentada

Nueve tesis no cumplen con el análisis, uno si lo hace 


\section{Cont... Cuadro 1}

\begin{tabular}{ll}
\hline $\begin{array}{l}\text { Las bases teóricas proporcionan fundamentación } \\
\text { conceptual actualizada }\end{array}$ & $\begin{array}{l}\text { Siete tesis si lo hacen, tres no responden a este re- } \\
\text { quisito }\end{array}$ \\
\hline $\begin{array}{l}\text { Se describen con claridad las teorías que sustentan } \\
\text { las categorías de investigación. }\end{array}$ & $\begin{array}{l}\text { Siete investigaciones no realizan esa descripción, tres } \\
\text { si lo hacen }\end{array}$ \\
\hline $\begin{array}{l}\text { Las teorías presentadas mantienen congruencia entre } \\
\text { sí }\end{array}$ & $\begin{array}{l}\text { Nueve trabajos no reflejan esa congruencia, uno si la } \\
\text { establece }\end{array}$ \\
\hline $\begin{array}{l}\text { Se evidencia la asunción de un punto de vista a partir } \\
\text { de los fundamentos teóricos }\end{array}$ & $\begin{array}{l}\text { Nueve tesis no evidencian selección de postura pro- } \\
\text { pia, una si lo hace }\end{array}$ \\
\hline $\begin{array}{l}\text { Se ubica el problema en un enfoque teórico determi- } \\
\text { nado }\end{array}$ & $\begin{array}{l}\text { Nueve trabajos no contextualizan el problema en al- } \\
\text { guna teoría, una lo hace acertadamente }\end{array}$ \\
\hline $\begin{array}{l}\text { Se retoman las teorías para explicar los datos ha- } \\
\text { llados }\end{array}$ & $\begin{array}{l}\text { Seis proyectos no logran esa integración, cuatro si lo } \\
\text { logra }\end{array}$ \\
\hline $\begin{array}{l}\text { Se presenta un texto coherente y cohesivo, responde } \\
\text { a las convenciones de la escritura. }\end{array}$ & $\begin{array}{l}\text { Ocho tesis trasgreden esas normas, dos lo hacen } \\
\text { adecuadamente }\end{array}$ \\
\hline Las fuentes son citadas correctamente & $\begin{array}{l}\text { Cinco lo hacen de forma regular, cuatro registra las } \\
\text { fuentes incorrectamente, uno lo hace acertadamente }\end{array}$ \\
\hline
\end{tabular}

Fuente: Elaboración propia

Los siguientes resultados son del análisis de los antecedentes de la investigación, mediante las categorías seleccionadas:

a) Las reseñas de estudios relacionadas con las variables de la investigación, se puede observar que todas las tesis si responden a esta exigencia, así como incluyen el número de antecedentes exigidos; b) Se establece clara vinculación y pertinencia entre los antecedentes y el problema. La mayoría de los trabajos analizados no logran precisar claramente la necesaria relación entre los antecedentes y la investigación; c) Los antecedentes son relevantes para el problema de investigación. En todos los trabajos los estudios previos tienen pertinencia con el tema en estudio; d) Las investigaciones presentadas son actualizadas. La mayoría de las tesis presentan antecedentes cuya vigencia data de cinco años.

Como se puede observar de las cuatro categorías evaluadas para los antecedentes, las tesis analizadas se ajustan satisfactoriamente a tres de ellas, ello significa que de modo acertado se reconstruye el fenómeno en estudio desde la visión de otros para orientar la perspectiva particular que el investigador adoptará.

Sobre los resultados para las Bases Teóricas, el contenido de las teorías permite explicar el objeto de estudio. Seis trabajos presentan contenidos que guardan alguna relación con la investigación, pero no se logra una explicación satisfactoria del objeto de estudio. Mientras que cuatro de ellas si lo hacen adecuadamente, en este sentido se obtuvo lo siguiente: a) Se evidencia análisis crítico de la información presentada. En nueve trabajos no hay análisis crítico, sólo uno responde a este requerimiento; b) Las bases teóricas proporcionan fundamentación conceptual actualizada. Siete tesis sí 
presentan teorías actualizadas, solo tres refieren principios teóricos considerados desactualizados; c) Se describen con claridad las teorías que sustentan las categorías de investigación. En siete trabajos no hay claridad teórica para explicar las categorías, las tres restantes si lo hacen adecuadamente; d) Las teorías presentadas mantienen congruencia entre sí. En nueve trabajos no se observa congruencia evidente entre las teorías, solo uno lo logra; e) Se evidencia la asunción de un punto de vista a partir de los fundamentos teóricos. Nueve tesis no presentan la escogencia de puntos de vista particulares considerando las teorías, solo una evidencia esta característica, f) Se ubica el problema en un enfoque teórico determinado. Nueve trabajos no responden a este criterio, solo una se ajusta adecuadamente.

Se retoman las teorías para explicar los datos hallados. Seis trabajos no logran interpretar los datos apoyándose en las teorías, cuatro si lo hacen. Se presenta un texto coherente y cohesivo, responde a las convenciones de la escritura. Ocho trabajos no logran presentar un texto adecuado a las exigencias de la escritura, dos si lo hacen. Las fuentes son citadas correctamente. Cinco trabajos citan las fuentes referenciales de modo regular, cuatro lo hacen incorrectamente, una lo realiza acertadamente. De las diez categorías de análisis consideradas para las bases teóricas, nueve fueron evaluadas negativamente, ello indica que estos estudiantes no lograron desarrollar organizada y sistemáticamente el conjunto de principios, conceptos, ideas y teorías que permiten apoyar la investigación, así como no pudieron comprender la perspectiva o enfoque desde el cual el investigador inicia, y a través del cual da sentido a sus hallazgos.

\subsection{Caracterización de los antecedentes}

En cuanto a las tres primeras categorías que aluden a los antecedentes de la investigación relativas a: se presentan reseñas de estudios relacionadas con las variables de la investigación, se establece clara vinculación entre los antecedentes y el problema y los antecedentes son relevantes para el problema de investigación, se puede precisar lo siguiente: todos los trabajos analizados presentan estudios en los que previamente se han abordado las categorías que integran el problema de investigación. Lo cual es fundamental ya que el proceso investigativo por su naturaleza reclama conocer hasta dónde se ha avanzado en determinado campo disciplinar, y los nuevos conocimientos se cimentan sobre los ya existentes.

Por otro lado, se puede observar que el total de las investigaciones presentan antecedentes relacionados con las categorías en estudio. De acuerdo con las normativas de la UPEL se deben citar un mínimo de cuatro a cinco estudios previos.

Es pertinente señalar que vincular, de modo explícito, los antecedentes con el problema es una necesidad pues ello implica establecer referencia con otros modos de encarar el mismo tópico de interés, valorar los aportes de otros, analizar si los métodos pueden ser válidos para el estudio actual, establecer la factibilidad de usar los mismos instrumentos, tipo de sujetos, forma de recolectar los datos, procedimientos para analizar los datos, comparar la similitud y diferencias de los resultados 
obtenidos por otros con respecto a los que están en discusión (Sánchez, 2014, Hernández, et al, 2014, García y Andrade, 2014, Arias, 2012).

En este aspecto, aunque se hace un esfuerzo por vincular antecedentes con problema, en general, se establece una relación superficial que se limita a hacer un comentario básico el cual, la mayoría de las veces, apunta a señalar que las conclusiones del antecedente le aportan para la implementación de la propuesta, pero no explican cuáles aspectos de esas conclusiones son pertinentes para el estudio. No se evidencia que haya verdadera comprensión sobre cuáles son las implicaciones que los antecedentes tienen para el nuevo estudio para de este modo apropiarse de una perspectiva teórica de inicio.

En el siguiente caso, cuando el estudiante quiere relacionar uno de los antecedentes con su trabajo solo hace un comentario elemental que no involucra ningún análisis.

"...se puede indicar que la investigación aporta al presente estudio datos y conclusiones que contribuyen a sustentar la necesidad de generar acciones que contribuyan a estimular en los docentes la puesta en práctica de los Proyectos de aprendizaje" (Balbuena,2016).

Otra manera de intentar realizar esa correlación es tomar textualmente un concepto relevante del antecedente, como se ve en el siguiente caso:

Esta investigación presenta pertinencia con el estudio debido a que refiere aspectos coincidentes con la investigación que se realiza tales como que "los proyectos de aprendizaje son unidades de planificación que de ser bien planeados deben contribuir a elevar la calidad educativa...". (Balbuena,2016)

En contraste con estas falencias, la tesis que elabora el marco teórico de manera acertada permite observar un ejemplo de cómo se establece la relación entre antecedentes y problema de modo adecuado.

Dicha investigación constituye uno de los antecedentes más relevantes para el presente trabajo, ya que se aborda y se ve a la lengua desde un punto de vista mucho más amplio, sin dejarse atrás los viejos paradigmas de enseñanza de lenguas, como lo son la gramática tradicional y la traducción, pero a su vez toma en cuenta el entorno a la hora de enseñar una lengua extranjera (Maldonando,2014)

Son análisis necesarios para crear vinculaciones entre los antecedentes, bases teóricas y su relación con el objeto de la investigación. Los investigadores serán los responsables de establecer y dejar con claridad la redacción de las vinculaciones necesarias entre estas categorías de análisis.

\subsection{Caracterización de las bases teóricas}

Para la categoría el contenido de las teorías permite explicar el objeto de estudio, se obtuvo que, en general, las tesis analizadas desarrollan aspectos teóricos sobre las categorías estudiadas, pero es un planteamiento que se limita a compendiar los aportes de distintos autores, lo que en muchas ocasiones se hace copiando textualmente las ideas originales. Es así que está ausente el desarrollo y adopción del punto de vista particular que explica el problema, lo que corrobora Guerra (2016:178) al afirmar que construir la postura epistemológica necesita que la mirada trascienda "el análisis acumulado de conocimientos para brindar una perspectiva de nuevos sentidos.

En el siguiente ejemplo el comentario solo repite las ideas 
originales de las fuentes citadas:

"...La formulación de las estrategias incluye la identificación de las debilidades y fortalezas internas de una organización, la determinación de las oportunidades y amenazas externas de una institución, la fijación de objetivos, el desarrollo de estrategias alternativas, el análisis de dichas alternativas y la decisión de cuales escoger". (Pereira, 2015)

El análisis crítico de la información presentada, evidencia que lo crítico es escaso porque no se muestra la interpretación personal que el investigador debería hacer de las teorías en discusión, es decir, hay muy pocas comparaciones, argumentaciones, inferencias, deducciones, razonamientos, explicaciones y críticas.

Las bases teóricas proporcionan fundamentación conceptual actualizada. La mayoría de los trabajos analizados sí cuentan con fundamentación conceptual actualizada y las fuentes citadas tienen promedio de cinco años de actualidad, lo cual es comprensible dado que es un requisito de la UPEL citar fuentes de reciente publicación, pues ello debería garantizar que los trabajos de grado recopilen el conocimiento más novedoso. De acuerdo con Sánchez, (2014), cumplir esa exigencia es determinante porque así se sabe si la idea inicial tiene sentido en el contexto de los saberes actuales.

La descripción de las teorías que sustentan las categorías de investigación, se realizan mediante los datos muestran que en siete trabajos solo se enuncian algunos elementos que pueden guardar alguna relación con las categorías analizadas. No se observa la necesaria red de conceptos y definiciones coherentes entre sí y con el tema en estudio.

La asunción de un punto de vista a partir de los fundamentos teóricos. Se ubica el problema en un enfoque teórico determinado. No se asumen posturas acerca de los aspectos teóricos presentados, no se comprueba que haya discernimiento acerca de si se escoge una teoría desarrollada que explica el problema, si se seleccionan varias teorías 0 a partir de varias generalizaciones empíricas (González, 2015); en consecuencia, tampoco el investigador elabora su propia perspectiva teórica ni ubica el problema en una teoría puntual.

Logran asumir puntos de vista y tener claridad para ubicar el estudio en una teoría puntual, resulta difícil para la mayoría de estos tesistas ya que su nivel de comprensión de las teorías citadas es limitado, por lo cual es común encontrar abundantes citas textuales con comentarios interpretativos incoherentes, como se puede comprobar en el siguiente ejemplo. Sobre elementos de eficacia, para el autor Serna (2003). "la eficacia mide el grado en que se alcanzaron los objetivos o metas de una organización, sin tener en cuenta los recursos empleados. Se expresan en términos cuantitativos, pero no miden la eficiencia en la utilización de los recursos" (p258). Es decir, la eficacia busca armonizar la organización con todas las condiciones" (T3V2015).

Se retoman las teorías para explicar los datos hallados. En la mayoría de los trabajos analizados se observan intentos por explicar los datos hallados usando las teorías referidas, sin embargo, no se concreta de modo acertado. La tendencia es a señalar los datos estadísticos obtenidos para algún indicador o citar un concepto que alude a ese indicador, pero no se logra el análisis requerido que explique el dato mediante el principio teórico. En relación a las 
respuestas obtenida se establece que los docentes no organizan actividades que fomenten la participación, pero a su vez esta dispuestos a colabora en esos procesos una vez que sean entrenados en la forma de hacerlo. En este sentido, Sirvent (2010:15) explica que la participación, cooperación y la organización de la comunidad escolar es algo primordial, por ello el docente en el Deber ser, tiene que fomentarlas en los miembros (que la conforman), y los organismos que hacen vida de forma directa o indirecta en la sociedad y el medio que rodea a los planteles y centros educativos (T4G2016).

Por el contrario, el trabajo T2G2016 al retomar teorías para explicar hallazgos refiere lo planteado por el autor pionero de la lingüística funcional (Halliday) y explica sus datos propios haciendo inferencias a partir de esos principios:

Esta visión del idioma es pilar fundamental de la presente investigación, puesto que hoy en día en las aulas venezolanas se vivencia todo lo contrario: profesores que enfocan el idioma desde una perspectiva disgregada, teniendo una visión pobre y muy corta, esta situación solo permite que los estudiantes memoricen vocablos $y$ frases hechas con tal de aprobar la materia (Maldonado, 2014).

Se presenta un texto coherente y cohesivo, responde a las convenciones de la escritura. En la mayoría de los trabajos hay debilidades al respecto; las fallas principales tuvieron que ver con la transgresión de las reglas que aportan cohesión y coherencia, éstas apuntan hacia la estructura que forma parte de las oraciones y párrafos que integran un texto y que a través de mecanismos de cohesión (sustitución, elipsis, deixis textual, proformas lexicales $y$ gramaticales) establecen las relaciones intratextuales y se convierten en un tipo de guía para que el lector pueda inferir los significados presentes en un texto (Flower y Hayes 1996).

Este ejemplo muestra total incoherencia y transgresión de normas básicas textuales.

En otras palabras la convivencia escolar es una actividad en la que el ser humano se enfrenta a diario, en la manera es que los directivos y docentes le toca vivir en cada espacio donde se relaciona con los miembros de la comunidad escolar, donde se menciona lo siguiente: (Sánchez, 2016)

También habría que agregar, el uso inapropiado de muchas convenciones de la escritura como signos de puntuación, acentuación, reglas ortográficas, entre otras. Lo cual es contrario a la necesidad de que el trabajo de grado se ajuste al registro estándar y formal (Arnáez, 2014). Este último hallazgo coincide con el de García y Andrade (2014), quienes corroboraron esta misma debilidad en tesis de posgrado con mención publicación.

En este ejemplo se puede observar que el tesista al incorporar algún comentario de su autoría rompe con el estilo coherente que traía el texto, se observa ambigüedad en el significado y se rompe la cohesión textual.

En el presente, se encuentra a diferentes autores que se han tomado la tarea de hacer aportes significativos para la conceptualización de orientación y todos ellos se puede apreciar como lo alude Senta E. (1979: 169) citada por Monroy Y. (2000), al referirse a la orientación como: "Un proceso o conjunto de acciones para ayudar a otros en la solución de situaciones críticas y conflictivas o en la satisfacción de necesidades para el logro de un estado de bienestar". De la misma manera, aquí, el investigador 
se basó en las concepciones ofrecidas por autores relevantes procurando acercarse al concepto esencial de la orientación (Ovalles, 2011).

Se puede condensar lo dicho hasta aquí con respecto a las bases teóricas, precisando que ellas, en general, son tomadas de los autores originales sin hacer mayores cambios, así abundan las citas textuales. Esto se corrobora cuando se perciben grandes diferencias discursivas entre el marco referencial y el resto de los capítulos del trabajo. Desde luego que esa debilidad se convierte en un problema por cuanto el marco referencial debe ser un texto que refleja las particularidades del autor ya que es el producto de su interpretación, de la confluencia de sus conocimientos previos con los nuevos, de la comprensión propia del fenómeno evidenciada en la escogencia de una teoría de abordaje.

Más aun, está ausente la conclusión global necesaria al finalizar cada tópico, ello constituye el aporte del investigador, tampoco se presentan explicaciones que correlacionen los temas. Estos textos se desarrollan mediante apartados o estancos sin cohesión, de los cuales se infiere cierta relación porque abordan aspectos que tratan de la problemática en estudio.

Las fuentes son citadas correctamente, en la misma se observa en la mayoría de los trabajos un uso irregular de las normas para citar el aparato crítico. Las debilidades al respecto son: aparece mucha información tomada de otras fuentes y no se reseñan los autores, cuando se parafrasea no se indican los autores de donde se toman las ideas, no se hace uso de los distintos tipos de citas, predominan las citas textuales, estas últimas si se registran apropiadamente.
El ejemplo siguiente permite observar que se registran los datos para una cita textual de modo inapropiado. Por su parte, Pereira (2015) afirma que la evaluación institucional constituye un proceso dinámico, continuo, permanente e inminente a toda institución"

El inadecuado tratamiento del discurso ajeno (Beke, 2011) evidenciado en un uso muy precario de las citas limita considerablemente el desarrollo de los temas, dado que la información que estas reportan permite profundizar en los conocimientos explorados, así como ofrecen la posibilidad de citar datos actualizados sobre la temática en discusión. Es indudable que la habilidad para emplear acertadamente las citas no se ha consolidado, lo cual es muy negativo pues su empleo acertado representa un ejemplo de lo que son los estilos de pensamiento y de razonamiento académico, concretados mediante determinadas convenciones discursivas.

\section{Reflexiones finales}

La interpretación de las características del marco teórico referencial de los trabajos de grado analizados en las áreas de Gerencia Educativa y Educación para la gestión comunitaria, permitió concluir lo siguiente:

En cuanto a los antecedentes sí se citan referencias actualizadas, pero no se logran relacionar apropiadamente con el interés investigativo, se evidencia una comprensión mecanicista y limitada del conocimiento que impide se integre una postura teórica de inicio.

Por lo que se refiere a las bases teóricas, predomina como característica el que los tesistas se limitan a exponer las teorías sin el respectivo análisis, no 
establecen la perspectiva o enfoque que facilita explicar el objeto investigativo y a través del cual deberán interpretar sus hallazgos. En consecuencia, están ausentes las nuevas apropiaciones que se logran mediante la interpretación, análisis y síntesis de conceptos, teorías, leyes y categorías, de modo que tampoco se pueden relacionar los datos empíricos con las teorías, no pudiéndose justificar las inferencias necesarias que cerrarían el ciclo investigativo.

En general, hay transcripción de aspectos teóricos informativos sobre el tema en estudio, predomina un distanciamiento de esas premisas y de lo que cree el investigador, pues el texto no evidencia la interrelación necesaria entre teorías y problema.

Todavía cabe señalar las debilidades en el manejo de competencias textuales para construir en texto coherente y cohesivo que se debe caracterizar por un alto nivel de complejidad y de exigencia en su construcción discursiva y conceptual. Así como se evidenció un manejo rudimentario de citas y referencias, por lo que existen dificultades para reportan las voces de otros, por ende, para manejar el código en el que se comunican los conocimientos científicos.

Por último, hay una evidente dificultad para presentar el marco teórico como conceptos, integrados por otros conceptos más simples, ordenados y unidos que logren una agrupación para derivar unos de otros, de modo tal que se construya una red significativa, coherente entre sí y con un referente común. Por lo tanto, si el marco teórico es inadecuado o insuficiente, la respuesta al problema, aun cuando éste haya sido bien planteado, será inadecuada, insuficiente o falsa.

\section{Referencias Bibliográficas}

Arias, Fidias (2012), El Proyecto de Investigación: Introducción a la Metodología Científica. Caracas: Episteme.

Arnáez, Pablo (2014), Propuesta pedagógica: la secuencia didáctica y el proyecto de trabajo de grado.En $\mathrm{S}$. Serrano y R. Mostacero. (Comp.). La escritura académica en Venezuela. Investigación, reflexión y propuestas (pp. 307-327).Mérida: Universidad de Los Andes.

Balbuena, Grecia (2016), Acciones gerenciales centradas en la planificación estratégica para el fortalecimiento de los proyectos de aprendizaje. Trabajo de grado de maestría no publicado. Universidad Pedagógica Experimental Libertador. Mérida, Venezuela

Beke, Rebeca (2011), El conocimiento ajeno en las disciplinas. Referencias y citas. En Adriana Bolívar y Rebeca Beke. (Comp.) Lectura y escritura para la investigación. (pp.131-139). Caracas: Universidad Central de Venezuela.

Bolívar, Adriana y Beke Rebeca (Comp.) (2011), Lectura y escritura para la investigación. Caracas: Universidad Central de Venezuela.

Castañeda, Ileana (2014), El marco metodológico en la investigación en salud con enfoque de género. Revista Cubana de Salud Pública, 40(2), 249-257.

Chacín, Migdy, Cobos, Leocadia y Aray, María (2009), Los colectivos de investigación: una propuesta para integración de saberes comunidad - universidad. Universidad Nacional Experimental Simón Rodríguez (UNESR). Disponible para Internet http://www.ucv.ve/fileadmin/user upload/vrac/documentos/Curricular_ 
Documentos/Evento/Ponencias_1/ Chacin_Cobos_y_Aray.pdf [Consultado el $\overline{15}$ de junio de 2018]

Flower, Linda y Hayes, Jhon (1996), La teoría de la redacción como proceso cognitivo. Textos en contexto 1. Los procesos de lectura y escritura. Lectura y Vida, Asociación Internacional de Lectura

Frances, Erlich y Shiro, Martha (2011), La argumentación en el discurso académico. En A. Bolívar y Rebeca Beke. (Comp.) Lectura y escritura para la investigación. (pp.163-184). Caracas: Universidad Central de Venezuela.

García, Marisol y Andrade, Ender (2014), Trabajos especiales de grado con Mención Publicación. Caso: Especialización en promoción de la Lectura y Escritura. En S. Serrano y R. Mostacero. (Comp.). La escritura académica en Venezuela. Investigación, reflexión y propuestas. (pp. 165-194).Mérida: Universidad de Los Andes.

Gómez, Rafael (2016), Actividades gerenciales para la promoción de los valores sociales en los estudiantes. Universidad Pedagógica Experimental Libertador. Mérida, Venezuela.

González, José (2015), Modelos y marcos teóricos en la investigación en pensamiento numérico en España. En C. Fernández, M. Molina y N. Planas (eds.), Investigación en Educación Matemática XIX (pp. 21-37). Alicante: SEIEM.

Guevara, Ragnhild (2016), El estado de arte en la investigación: ¿análisis de los conocimientos acumulados o indagación por nuevos sentidos? FOLIOS, 44, 165-179.

Hernández, Roberto, Fernández, Carlos y Baptista, María (2014), Metodo- logía de la investigación. México: Mac Graw Hill.

Martínez, Miguel (2009), Epistemología y metodología cualitativa en las Ciencias Sociales. México: Trillas.

Ovalles, Angie. (2011), Propuesta sustentada en la orientación personal para la elaboración de objetos recreativos mediante la reutilización de desechos sólidos que optimicen el funcionamiento del parque de la institución. Trabajo de grado de maestría no publicado. Universidad Pedagógica Experimental Libertador. Mérida, Venezuela

Pereira, Edilmar (2015), Habilidades gerenciales para promover el desarrollo organizacional en los docentes de Educación Media. Trabajo de grado de maestría no publicado. Universidad Pedagógica Experimental Libertador. Mérida, Venezuela

Pino, Ricardo (2009), Marco Teórico Referencial de la Investigación. Concepto y funciones. Revista Varela. 24(9), 1-17.

Pino, Ricardo (2009), Marco Teórico Referencial de la Investigación. Concepto y funciones. Revista Varela. 24(9), 1-17.

Reidil-Martínez, Lucy (2012), Marco conceptual en el proceso de investigación. ELSEVIER, 1(3): 146

Sánchez, Carlos (2014), El estado del conocimiento como estrategia para la elaboración de trabajos recepcionales en posgrado. Ponencia presentada durante el Congreso Internacional de Evaluación Educativa: Tlaxcala: Universidad Autónoma de Tlaxcala.

Sánchez, Manuel (2014), El marco teórico como herramienta conceptual de investigación científica aplicada a la investigación jurídica. Revista Tele- 
Producción de conocimiento teórico: El caso de Universidad Pedagógica Experimental Libertador (Venezuela)

Teresa de Jesús Molina Gutiérrez; Gisela Quintero y Lenin Horacio Burbano García

mática de Filosofía del Derecho, 17, pp. 83-109.

Sánchez, Mireya (2016), Formación ética profesional para el mejora- miento del desempeño gerencial del docente en la Educación Media. Trabajo de grado de maestría no publicado. Universidad Pedagógica Experimental Libertador. Mérida, Venezuela.

- Esta obra está bajo una licencia de Creative Commons Reconocimiento-NoComercial- Compartirlgual 3.0 Unported. http://creativecommons.org/licenses/by-nc-sa/3.0/deed.es_ES 